\title{
Comparison of Novel Metabolic Indices in Estimation of Chronic Kidney Diseases in a Southern Chinese Population
}

This article was published in the following Dove Press journal: Diabetes, Metabolic Syndrome and Obesity: Targets and Therapy

\author{
Tong Chen ${ }^{1, *}$ \\ Xuan Wang ${ }^{1, *}$ \\ Xin Wang' \\ Haishan Chen' \\ Hua Xiao' \\ Hongjuan Tang' \\ Ling Feng' \\ Zhicong Xiang' \\ Hequn Zou ${ }^{1,2}$ \\ Xiaofei Shao' \\ 'Department of Nephrology, The Third \\ Affiliated Hospital, Southern Medical \\ University, Guangzhou 5I 0630, People's \\ Republic of China; ${ }^{2}$ Department of \\ Nephrology, Pinghu Hospital, Health \\ Science Center, Shenzhen University, \\ Shenzhen 518116, People's Republic of \\ China
}

*These authors contributed equally to this work
Background: To determine the optimal cut-off values and evaluate the associations of product of triacylglycerol and glucose (TyG), lipid accumulation product (LAPI), visceral adiposity index (VAI) with chronic kidney diseases (CKD) stratified by sex.

Methods: From January to April 2018, our team had conducted a large-scale cross-sectional survey that contained 2720 individuals on the southern coast of China. Logistic regression analysis and receiver operating characteristic (ROC) analyses were used to evaluate the optimal cut-off and value of TyG, LAPI, VAI for predicting CKD.

Results: A multivariate logistic regression analysis found that the TyG had the better value of prediction for the presence of CKD for the highest quartile vs the lowest quartile in both males (OR: 3.65; 95\% CI, 2.04-6.52; $\mathrm{p}<0.001$ ) and females (OR: 3.50; 95\% CI, 2.20-5.56; $<0.001$ ), followed by LAPI and VAI, when further adjusted for cofounder factors, LAPI and VAI both lost their independence, and only TyG remains its significant association with CKD in both males (OR: 2.81; 95\% CI, 1.25-6.30; $<<0.001$ ) and females (OR: 3.22; 95\% CI, 1.56-6.61; $<<0.001$ ). ROC curve showed that TyG had the highest AUC for predicting CKD in males (AUC: 0.618). TyG (AUC: 0.670) and LAPI (AUC: 0.670) both had the highest AUC in females. United predicted models which contain TyG were conducted for predicting CKD in males (AUC: 758) and females (AUC: 0.773) and results indicated that multivariate analysis of TyG and other traditional factors can impressively improve the accuracy of predictive probability for CKD.

Conclusion: TyG is a priority to the other two novel indices and may become valuable makers and have strong predictive power for predicting CKD, especially in females.

Keywords: product of triacylglycerol and glucose, lipid accumulation product, visceral adiposity index, chronic kidney diseases

\section{Introduction}

Chronic kidney diseases (CKD) have gradually become a worldwide public health burden on the world health-care system. According to previous research, the prevalence of CKD was $10.8 \%$ in China, and CKD is positively related to cardiovascular morbidity and mortality, especially in China. ${ }^{1-3}$ The cost which patients spend on dialysis treatment for end-stage kidney diseases (ESKD) is about $\$ 14,300$ per year, which increases the economic burden to the Country and Society. ${ }^{4}$ In developed countries, CKD contributed to the leading cause of death, which makes the prevention and control of CKD particularly necessary. ${ }^{5}$

It is well known that obesity is related to CKD, but the critical mechanism is not yet clear. Previous studies showed that insulin resistance may play an important role
Correspondence: Xiaofei Shao Department of Nephrology, The Third Affiliated Hospital, Southern Medical Republic of China Email 413151187@qq.com

Hequn Zou

No. I, Fuxin Road, Pinghu Street, Longgang District, Shenzhen 518116, People's Republic of China

Email Hequnzou@hotmail.com 
in the progress of renal impairment. ${ }^{6}$ The current precise diagnosed test for insulin resistance is the hyperinsulinemic euglycemic clamp test, which is expensive and complex, another diagnosed test, homeostasis model assessment for insulin resistance (HOMA-IR), is also inconvenient and unpracticable in clinical conditions because HOMA-IR needs patients' serum insulin level to estimate and calculate. ${ }^{7,8}$ Thus, novel and surrogate index of insulin resistance and obesity are particularly required.

Recently, three new indices, visceral adiposity index (VAI) and Triglycerides-glucose index (TyG), the lipid accumulation product index (LAPI), were proposed. These novel indices have been used as estimators of visceral adiposity dysfunction and lipid overaccumulation, ${ }^{9,10}$ and these novel indices have been found to correlate with insulin resistance, ${ }^{11}$ impaired fasting glucose, ${ }^{12}$ discrimination of prediabetes and diabetes, ${ }^{13,14}$ metabolic syndrome $^{15}$ and Chronic Kidney Disease. ${ }^{16,17}$ However, there are few studies to compare the different predictive value of three novel indices for $\mathrm{CKD}$, especially in the southern Chinese population, so our study aims to further explore which indices were superior to associate with CKD and identify individuals with CKD in the Chinese population.

\section{Materials and Methods Study Population}

From January to April 2018, this cross-sectional survey was based on a community random sample population conducted in Wanzhai Town, Zhuhai City, on the southern coast of China. The study was conducted in accordance with the Declaration of Helsinki and was approved by the Ethics Committee of the Third Affiliated Hospital of Southern Medical University. Written informed consent forms were signed by all participants. Participants were selected by multi-stage stratified random method. Firstly, our team selected two communities randomly from Wanzhai Town; secondly, from two selected communities, 500 families were randomly chosen as the sample family; and finally, we included and sampled all the residents aged from 18 to 75. According to this method, a total of 2720 participants completed the survey. The exclusion criteria included: missing essential information, like gender; education; disease history; lifestyle information; blood pressure (BP), waist measurement; blood glucose and cholesterol, triglyceride (TG) levels information.

\section{Data Collection}

The participants had finished the Questionnaires which included data on gender, age, the degree of education, drug medication, smoking situation, alcohol use, physical activity frequency and other sociodemographic data of volunteers. After completing the questionnaires, volunteers had rest for at least 5 minutes, and the blood pressure and Waist circumference were immediately measured. The blood pressure of the left arm was measured using a mercury desk-top sphygmomanometer, and the mean blood pressure was calculated after three records were taken by the sphygmomanometer. Waist circumference was measured at the midway between the lower rib edge of the midline and the top of the hip bone. All blood samples were collected from the anterior cubital vein after $12 \mathrm{hrs}$ of fasting overnight and at the same time, fresh morning urine samples were collected. Blood samples collected in EDTA tubes containing inert separation gels were used to analyze the blood biochemistry. All blood samples were shipped to the testing central laboratory of the Third affiliated Hospital of Southern Medical University and analyzed within three hours. Serum creatinine was measured by enzymatic method, fasting blood glucose was measured in the same way (Roche Cobas 6000, Orion), the blood lipid paraments such as serum triglycerides (TG), serum total cholesterol (TC) and serum HDL-C, were tested by a colorimetric method (Roche Cobas 6000). After morning urine collection and stored at $-80^{\circ} \mathrm{C}$ for less than one week, urinary albumin was measured by immunoturbidimetric tests (Audit Diagnostics, Cork, Ireland) and urinary creatinine was evaluated through Jaffe's kinetic method (Audit Diagnostics, Cork). The urinary albuminto-creatinine ratio (ACR) value was calculated by the records of urinary albumin and urinary creatinine concentration.

\section{Definitions of VAI, LAPI and TyG Score}

The VAI score was calculated as followed. ${ }^{9}$ (1) In male population, $\mathrm{VAI}=[\mathrm{WC} /(39.68+1.88 \times \mathrm{BMI})] \times(\mathrm{TG} /$ $1.03) \times(1.31 / \mathrm{HDL}) .(2)$ In Females, the VAI $=[\mathrm{WC} /$ $(36.58+1.89 \times \mathrm{BMI})] \times(\mathrm{TG} / 0.81) \times(1.52 / \mathrm{HDL})$.

Product of triacylglycerol and glucose (TyG) was calculated according to published formula: $\mathrm{TyG}=\ln$ [fasting $\mathrm{TG}(\mathrm{mg} / \mathrm{dL}) \times$ FPG $(\mathrm{mg} / \mathrm{dL}) / 2]{ }^{18}$

The formula of LAPI: (1) males: LAPI $=(\mathrm{WC}-65)$ $\times \mathrm{TG}$; (2) Females: LAPI $=\left(\right.$ WC-58) $\times \mathrm{TG}^{19}$ 


\section{Definitions of CKD}

The CKD was diagnosed by following criteria: estimated glomerular filtration rate $(\mathrm{eGFR})<60(\mathrm{~mL} / \mathrm{min}$ per 1.73 $\mathrm{m} 2$ ) or urinary albumin-to-creatinine ratio (ACR) $>30 \mathrm{mg}$ / g. A formula from the Chinese-Modification of Diet Renal Disease (C-MDRD) study was used to calculate the estimated glomerular filtration rate (eGFR): GFR $(\mathrm{mL} / \mathrm{min} /$ $1.73 \mathrm{~m} 2)=175 \times(\mathrm{Scr})^{-1.234} \times(\text { Age })^{-0.179} \times($ if female,$\times$ $0.79) .{ }^{20,21}$

\section{Statistical Analysis}

SPSS software (version 20) was used to analyze data and a two-tailed p-value $<0.05$ was considered statistically significant. Normally distributed continuous variables were expressed as mean (standard deviation) and the $t$-test was performed to compare the differences of means between the groups. Continuous variables that did not show normal distribution would be presented as Median and interquartile range and the Wilcoxon test was used for comparison of differences in the medians. The categorical variables were expressed as absolute and percentiles or proportion. The chi-square $(\chi 2)$ test was conducted to compare the categorical variables. Binomial Logistic regression models were performed to assess the relationship between CKD and the three novel indicators of visceral adiposity index (VAI), Triglycerides-glucose index (TyG), the lipid accumulation product index (LAPI). To amplify the association between novel indicators and CKD, both males and females were transformed into four groups based on the quartiles of VAI, TyG, LAPI in the binomial logistic regression analysis. Subjects in quartile one considered as the reference group. A two-sided test in which a P-value of 0.05 was considered to be a statistically significant level was used. Two models were generated: Model1 adjust for age, education, physical activity, smoking, drinking; model two adjust for age, education, physical activity, smoking, drinking, systolic pressure, diastolic pressure, high-sensitivity $\mathrm{C}$ reactive protein, VLDL-C, LDL-C, TC. A receiver operating characteristic (ROC) curve analysis which was quantified by the area under the ROC curve (AUC) was used to evaluate the value of the novel indicators for predicting CKD by comparing the area under the ROC curve (AUC). The optimal cutoff points of those three new indicators were determined by Youden's index which can be calculated as (sensitivity +specificity-1).

\section{Results}

A total of 2720 participants (1002 males and 1718 females) were included in this study, the prevalence of CKD was $16.97 \%$ in males and $15.60 \%$ in females. The basic characteristics of the participants are shown in Table 1. According to Table, in males, the mean age and education status, history of hypertension and diabetes, physical inactivity were higher in CKD patients. The females showed the same results compared to the subjects without CKD. For anthropometric measurements, both males and females with CKD had lower height together with higher weight, waist circumference (WC), body mass index (BMI), systolic blood pressure, diastolic blood pressure. Additionally, for laboratory assays, significant differences were found in fasting plasma glucose (FPG), triglyceride (TG), total cholesterol, high-density lipoprotein (HDL), low-density lipoprotein (LDL), very-lowdensity lipoprotein (VLDL), hypersensitive C-reactive protein (hs-CRP) in patients with $\mathrm{CKD}$, furthermore, ACR, LAPI, TyG, VAI also were larger than subjects without CKD, while eGFR was in a lower level in CKD subjects. Unexpectedly, this study did not find a significant association between current smoking and drinking with CKD. Individuals may have changed their lifestyle after being diagnosed with CKD.

Multivariate logistic regression models were performed to analyze the association between three novel indices (LAPI, VAI, TyG) quartiles with CKD (Table 2). After adjustment for age, education, physical activity, smoking, drinking (Model 1), males in the 4th of LAPI, TyG, VAI had 2.87-, 3.65-, 2.50-fold increased risk of CKD than those in the 1st quartiles $(\mathrm{P}<0.001)$. Females in the 4th of LAPI, TyG, VAI had 2.45-, 3.50-, 2.13-fold increased risk of CKD. While further controlling for BMI, waist circumference, systolic pressure, diastolic pressure, highsensitivity $\mathrm{C}$ reactive protein, HDL-C, VLDL-C, LDL-C, TC, only TyG had significant ORs of the presence of CKD (OR: 2.81, 95\% CI, 1.25-6.30). In females, TyG also had the higher ORs (OR: 3.22, 95\% CI, 1.56-6.61) than other novel indices when comparing the top quartile with the bottom quartile. In total, TyG showed the largest association with CKD in both males and females in the multivariable-adjusted model. (Model 2)

The relative abilities of three novel indexes to identify patients with CKD are compared in Table 3. The abilities of novel indices for predicting CKD were the same in both genders. TyG had the highest $\mathrm{AUC}(\mathrm{AUC}=0.618)$ in three 
Table I Baseline Characteristics of the Study Population

\begin{tabular}{|c|c|c|c|c|c|c|}
\hline \multicolumn{4}{|l|}{ Males $(n=1002)$} & \multicolumn{3}{|l|}{ Females $(n=|7| 8)$} \\
\hline Parameters & $\begin{array}{l}\text { CKD (-) } \\
(n=832,83.03 \%)\end{array}$ & $\begin{array}{l}\text { CKD (+) } \\
(n=170,16.97 \%)\end{array}$ & $\mathbf{P}$ & $\begin{array}{l}\text { CKD (-) } \\
(n=\mid 450,84.40 \%)\end{array}$ & $\begin{array}{l}\text { CKD (+) } \\
(n=268,15.60 \%)\end{array}$ & $\mathbf{P}$ \\
\hline Age (years) & $54.0(14.4)$ & $63.9(12.6)$ & $<0.001$ & $52.7(13.7)$ & $63.6(13.4)$ & $<0.001$ \\
\hline Education (high school or above, $n(\%)$ ) & $395(47.5 \%)$ & 59 (34.7\%) & $<0.01$ & $512(37.0 \%)$ & $43(17.1 \%)$ & $<0.001$ \\
\hline History of hypertension, n (\%) & $204(24.5 \%)$ & $100(59.2 \%)$ & $<0.001$ & $280(19.3 \%)$ & $135(50.4 \%)$ & $<0.001$ \\
\hline History of diabetes, n (\%) & $56(6.7 \%)$ & $35(20.7 \%)$ & $<0.001$ & $93(6.4 \%)$ & $43(16.0 \%)$ & $<0.001$ \\
\hline Physical activity, n (\%) & $463(55.6 \%)$ & $101(59.4 \%)$ & 0.37 & $797(56.0 \%)$ & $173(64.8 \%)$ & 0.008 \\
\hline Current smoking, n (\%) & $270(32.5 \%)$ & $53(31.2 \%)$ & 0.75 & $13(0.9 \%)$ & I (0.4\%) & 0.39 \\
\hline Current alcohol use, $\mathrm{n}(\%)$ & $115(13.8 \%)$ & $25(14.7 \%)$ & 0.76 & $13(0.9 \%)$ & I (0.4\%) & 0.38 \\
\hline Height $(\mathrm{cm})$ & $166.66(6.52)$ & $165.30(5.96)$ & 0.012 & $|55.4|(5.86)$ & $153.06(6.59)$ & $<0.00$ I \\
\hline Weight (kg) & $67.18(10.39)$ & $68.69(10.94)$ & 0.088 & $56.68(8.59)$ & $58.75(9.95)$ & $<0.001$ \\
\hline Waist circumference $(\mathrm{cm})$ & $87.54(9.43)$ & $91.08(9.83)$ & $<0.001$ & $81.23(9.67)$ & $86.80(10.20)$ & $<0.001$ \\
\hline BMI $\left(\mathrm{kg} / \mathrm{m}^{2}\right)$ & $24.15(3.32)$ & $25.07(3.53)$ & $<0.001$ & $23.45(3.35)$ & $25.05(4.05)$ & $<0.001$ \\
\hline Systolic blood pressure $(\mathrm{mmHg})$ & $132.87(18.15)$ & $144.98(18.80)$ & $<0.001$ & 129.20 & |45.55 (2I.7I) & $<0.001$ \\
\hline Diastolic blood pressure $(\mathrm{mmHg})$ & $83.03(11.00)$ & $86.82(12.04)$ & $<0.001$ & $78.84(10.46)$ & $84.04(11.60)$ & $<0.001$ \\
\hline Fasting plasma glucose, $\mathrm{mmol} / \mathrm{L}$ & $5.16(1.18)$ & $5.95(2.24)$ & $<0.001$ & $5.06(0.96)$ & $5.84(1.85)$ & $<0.001$ \\
\hline Triglyceride, $\mathrm{mmol} / \mathrm{L}$ & $1.69(1.09)$ & $1.96(1.23)$ & $<0.001$ & $1.38(0.78)$ & $1.74(0.99)$ & $<0.001$ \\
\hline $\mathrm{HDL}-\mathrm{C}, \mathrm{mmol} / \mathrm{L}$ & $1.37(0.29)$ & $1.36(0.35)$ & 0.62 & $1.60(0.32)$ & $1.53(0.34)$ & $<0.001$ \\
\hline LDL-C, mmol/L & $3.11(0.90)$ & $3.23(1.00)$ & 0.13 & $3.20(0.91)$ & $3.45(0.98)$ & $<0.00$ I \\
\hline VLDL-c mmol/L & $0.76(0.47)$ & $0.89(0.56)$ & 0.005 & $0.62(0.35)$ & $0.78(0.44)$ & $<0.001$ \\
\hline Total cholesterol, mmol/L & $5.25(0.99)$ & $5.48(1.11)$ & $<0.01$ & $5.43(1.06)$ & $5.76(1.12)$ & $<0.001$ \\
\hline hypersensitive C-reactive protein, $\mathrm{mmol} / \mathrm{L}$ & $1.24(0.55,2.57)$ & $1.86(0.82,3.43)$ & $<0.001$ & I.I8(0.40,2.26) & $1.94(0.89,3.81)$ & $<0.001$ \\
\hline eGFR $(\mathrm{mL} / \mathrm{min} / 1.73 \mathrm{~m} 2)$ & $88.59(16.95)$ & $73,49(26.76)$ & $<0.001$ & $100.97(19.89)$ & $85.69(25.12)$ & $<0.001$ \\
\hline ACR (mg/g) & $7.42(4.77,11.35)$ & $44.88(30.48,87.47)$ & $<0.001$ & $9.77(6.71,14.67)$ & $48.60(34.99,90.83)$ & $<0.001$ \\
\hline LAPI $(\mathrm{cm} \cdot \mathrm{mmol} / \mathrm{L})$ & $40.42(33.90)$ & $53.73(41.80)$ & $<0.001$ & $34.27(27.33)$ & $52.40(36.76)$ & $<0.001$ \\
\hline VAI & $1.38(0.88,2.14)$ & $1.67(1.11,2.55)$ & $<0.001$ & $1.38(0.94,2.17)$ & $2.00(1.19,3.04)$ & $<0.00$ I \\
\hline TyG & $8.68(0.59)$ & $8.94(0.63)$ & $<0.001$ & $8.49(0.54)$ & $8.83(0.60)$ & $<0.001$ \\
\hline
\end{tabular}

Abbreviations: VAI, visceral adiposity index; LAPI, lipid accumulation product index; TyG, product of triacylglycerol and glucose; eGFR, the estimated glomerular filtration rate; $A C R$, urinary albumin-to-creatinine ratio.

novel makers in males, followed by LAPI (AUC $=0.607$ ), VAI (AUC=0.586) (Figure 1). The optimal cut-off value of TyG based on the Youden index was 8.74. TyG and LAPI both showed better accuracy for predicting CKD $(\mathrm{AUC}=0.670)$ than VAI $(\mathrm{AUC}=0.636)$ in females (Figure 2), the optimal cut-off value of TyG and LAPI were 8.59 and $37.49 \mathrm{~cm} * \mathrm{mmol} / \mathrm{L}$. Furthermore, the united models for predicting CKD were built in both males (including age, systolic pressure, high-sensitivity $\mathrm{C}$ reactive protein, $\mathrm{TyG}$ ) and females (including age, diastolic pressure, VLDL-C, high-sensitivity $\mathrm{C}$ reactive protein, TyG). The abilities of the united model for predicting CKD (males AUC: 0.753 and females AUC: 0.773) were stronger than a single novel indicator. For the three indices assessed, the AUC and Youden index of TyG, VAI, LAPI based on the ROC curve were all higher in females than males, which means these three novel indices were more significantly capable of predicting CKD in females than males.

\section{Discussion}

In this population-based cross-sectional study, we explored and analyzed the potential association between TyG, LAPI, VAI and CKD in the Chinese southern population. The main aim of this study was to investigate which surrogate maker was better to predict CKD. To our knowledge, there has been no relevant literature that confirms and compares a significant correlation between three novel indices and CKD so far. We found that TyG, LAPI, VAI all have a strongly positive relationship with CKD in both males and females. But only TyG and LAPI remained significant in males or TyG in females, after adjustment for blood pressure, high-sensitivity $\mathrm{C}$ reactive protein, VLDL-C, LDL-C, TC.

Recently, several previous epidemiologic studies have found that obesity and CKD were positively connected, especially visceral obesity (VAT). Obesity as a focus of growing attention has been associated with $\mathrm{CKD}$, even in 
Table 2 Multivariate Logistic Regression Analysis Showing Independent Predictors of CKD

\begin{tabular}{|c|c|c|c|c|c|c|c|c|}
\hline \multirow[t]{3}{*}{ Indices } & \multicolumn{4}{|l|}{ Males } & \multicolumn{4}{|l|}{ Females } \\
\hline & \multicolumn{2}{|l|}{ Model I } & \multicolumn{2}{|l|}{ Model 2} & \multicolumn{2}{|l|}{ Model I } & \multicolumn{2}{|l|}{ Model 2} \\
\hline & OR (95\% Cl) & $\mathbf{P}$ & OR (95\% Cl) & $\mathbf{P}$ & OR (95\% Cl) & $\mathbf{P}$ & OR (95\% Cl) & $\mathbf{P}$ \\
\hline \multicolumn{9}{|l|}{ LAPI } \\
\hline QI (reference) & I & & I & & I & & I & \\
\hline Q2 & $1.02(0.58-1.79)$ & 0.930 & $0.85(0.44-1.66)$ & 0.649 & $0.96(0.59-1.57)$ & 0.895 & $0.65(0.37-1.14)$ & 0.135 \\
\hline Q3 & $1.73(1.03-2.90)$ & 0.037 & $1.25(0.56-2.78)$ & $0.58 \mathrm{I}$ & $1.22(0.77-1.94)$ & 0.383 & $0.63(0.32-1.23)$ & 0.178 \\
\hline Q4 & $2.87(1.73-4.74)$ & $<0.001$ & $1.81(0.62-5.24)$ & 0.272 & $2.45(1.57-3.8 I)$ & $<0.001$ & $0.86(0.34-2.19)$ & 0.764 \\
\hline $\mathrm{P}$ for trend & & $<0.001$ & & 0.188 & & $<0.001$ & & 0.700 \\
\hline \multicolumn{9}{|l|}{ TyG } \\
\hline QI (reference) & I & & I & & I & & I & \\
\hline Q2 & $1.85(0.97-3.52)$ & 0.060 & $1.76(0.89-3.46)$ & 0.102 & $1.56(0.95-2.56)$ & 0.750 & $\mathrm{I} .37(0.8 \mathrm{I}-2.3 \mathrm{I})$ & 0.241 \\
\hline Q3 & $2.07(1.12-3.80)$ & 0.019 & $1.90(0.96-3.76)$ & 0.065 & $1.90(1.17-3.07)$ & 0.009 & $1.62(0.92-2.85)$ & 0.094 \\
\hline Q4 & $3.65(2.04-6.52)$ & $<0.001$ & $2.81(1.25-6.30)$ & 0.012 & $3.50(2.20-5.56)$ & $<0.001$ & $3.22(1.56-6.6 \mathrm{I})$ & 0.001 \\
\hline $\mathrm{P}$ for trend & & $<0.001$ & & 0.018 & & $<0.001$ & & 0.003 \\
\hline \multicolumn{9}{|l|}{ VAI } \\
\hline QI (reference) & I & & 1 & & I & & I & \\
\hline Q2 & $\mathrm{I} .40(0.8 \mathrm{I}-2.40)$ & 0.224 & I.3I(0.7I-2.49) & 0.371 & $0.82(0.49-1.35)$ & 0.442 & $0.64(0.36-1.12)$ & 0.123 \\
\hline Q3 & $2.20(1.33-3.62)$ & 0.002 & $1.92(0.95-3.86)$ & 0.067 & $1.49(0.94-2.37)$ & 0.089 & $0.95(0.5 I-1.76)$ & 0.872 \\
\hline Q4 & $2.50(1.51-4.14)$ & $<0.001$ & $2.01(0.75-5.36)$ & 0.164 & $2.13(1.37-3.32)$ & 0.001 & I.I I(0.49-2.52) & 0.797 \\
\hline $\mathrm{P}$ for trend & & $<0.001$ & & 0.089 & & $<0.001$ & & 0.473 \\
\hline
\end{tabular}

Note: Modell adjust for age, education, physical activity, smoking, drinking. Model2 adjust for age, education, physical activity, smoking, drinking, BMI, waist circumference, systolic pressure, diastolic pressure, high-sensitivity C reactive protein, VLDL-C, LDL-C, HDL-C, TC.

Abbreviations: VAI, visceral adiposity index; LAPI, lipid accumulation product index; TyG, product of triacylglycerol and glucose; CKD, chronic kidney disease.

Table 3 The Areas Under ROC Curve (AUC), Sensitivity and Specificity by the Optimized Cut-off Points for Novel Indices in Predicting CKD

\begin{tabular}{|l|l|l|l|l|l|}
\hline Risk Factors & AUC & Cut-Off Value & Youden' Index & Sensitivity & Specificity \\
\hline Male & & & & \\
VAI & $0.586(0.539-0.632)$ & 1.21 & 0.163 & 0.729 & 0.434 \\
TyG & $0.618(0.572-0.664)$ & 8.74 & 0.182 & 0.594 & 0.588 \\
LAPI & $0.607(0.560-0.654)$ & 36.83 & 0.211 & 0.635 & 0.576 \\
United predicted Model I & $0.753(0.713-0.792)$ & 0.22 & 0.398 & 0.598 & 0.800 \\
\hline Female & & & & $0.54 I$ & 0.654 \\
VAI & $0.636(0.600-0.673)$ & 1.88 & 0.228 & 0.686 \\
TyG & $0.670(0.636-0.705)$ & 8.59 & 0.275 & 0.598 & 0.676 \\
LAPI & $0.670(0.634-0.705)$ & 37.49 & 0.274 & 0.720 & 0.732 \\
United predicted Model 2 & $0.773(0.741-0.806)$ & 0.17 & 0.451 & \\
\hline
\end{tabular}

Notes: United predicted model I includes age, systolic pressure, high-sensitivity C reactive protein, TyG. United predicted model 2 includes age, diastolic pressure, VLDLC, high-sensitivity $C$ reactive protein, TyG.

Abbreviations: VAl, visceral adiposity index; LAPI, lipid accumulation product index; TyG, product of triacylglycerol and glucose; CKD, chronic kidney disease; AUC, area under the ROC (receiver operating characteristic) curve.

the early stage of mild renal function damage. ${ }^{22-24}$ Although the mechanisms that adiposity affect CKD are not entirely clear, insulin resistance may play a critical role in the progress of CKD. VAT is a hormonally active fat tissue that can take part in the progress of glucose metabolism. ${ }^{25,26}$ VAT also has been correlated with the decline of eGFR and type 2 diabetes. ${ }^{27-29}$ Accumulation of fat tissues in ectopic tissues may contribute to insulin resistance, compression of kidney, hypertension. ${ }^{30}$ Additionally, VAT also can induce obesity-related 


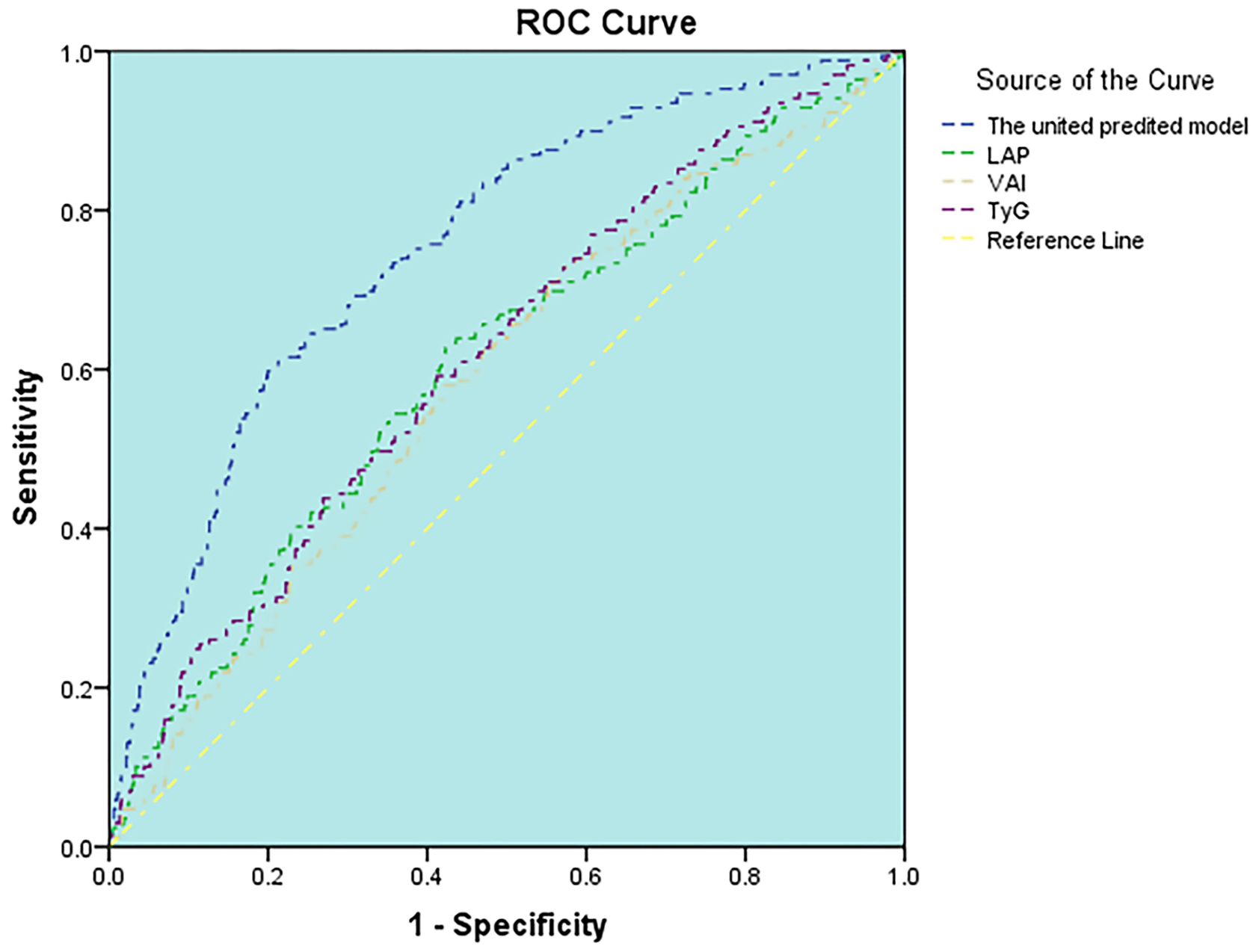

Diagonal segments are produced by ties.

Figure I ROC curve analysis of the value of TyG, LAPI, VAI, united predicted model for predicting CKD in males.

subclinical inflammatory changes, such as increased production of interleukin-6, tumor necrosis factor- $\alpha$ and C-reactive protein, which can precede the pathogenesis of $\mathrm{CKD}^{31}$ and abnormal activity of inflammation may result in disturbances in the insulin signaling pathway, inactivate insulin receptor substrate 1, insulin resistance $^{32,33}$ and interferences in kidney function and structure such as increased albuminuria. ${ }^{34}$ Many previous studies have shown that insulin resistance is an early metabolic complication and can occur in different stages or types of kidney diseases, such like the early stage of CKD, Immunoglobulin A nephropathy or polycystic kidney diseases. ${ }^{34-38}$

Accordingly, recognition of VAT and insulin resistance can have effective and clinical value to identify patients who have the potential risk of renal function damage. The current "gold standard" for measuring insulin resistance is hyper insulinemic euglycemic clamp test, which is inconvenient and expensive ${ }^{7}$ and researchers also proposed HOMA-IR to estimate insulin sensitivity, but HOMA-IR requires measurement of insulin, which is not always available. ${ }^{8}$ The current "gold standard" for quantifying VAT is image technique, such like CT and MRI, but the cost is too high to use in the clinical settings and the radiation exposure is another disadvantage of image technique. ${ }^{39}$ In this condition, simple and conveniently surrogate indices of VAT and insulin resistance are needed. The three novel metabolic indices VAI, LAPI, TyG have been proposed as markers of visceral adipose function and insulin resistance ${ }^{9,40}$ and have been linked to cardiometabolic diseases, ${ }^{9}$ impaired fasting glucose, ${ }^{12}$ discrimination of prediabetes and diabetes, ${ }^{13,14}$ metabolic syndrome ${ }^{15}$ and Chronic Kidney Disease ${ }^{16,17}$ in a cross-sectional study. VAI which combines both anthropometric and metabolic 


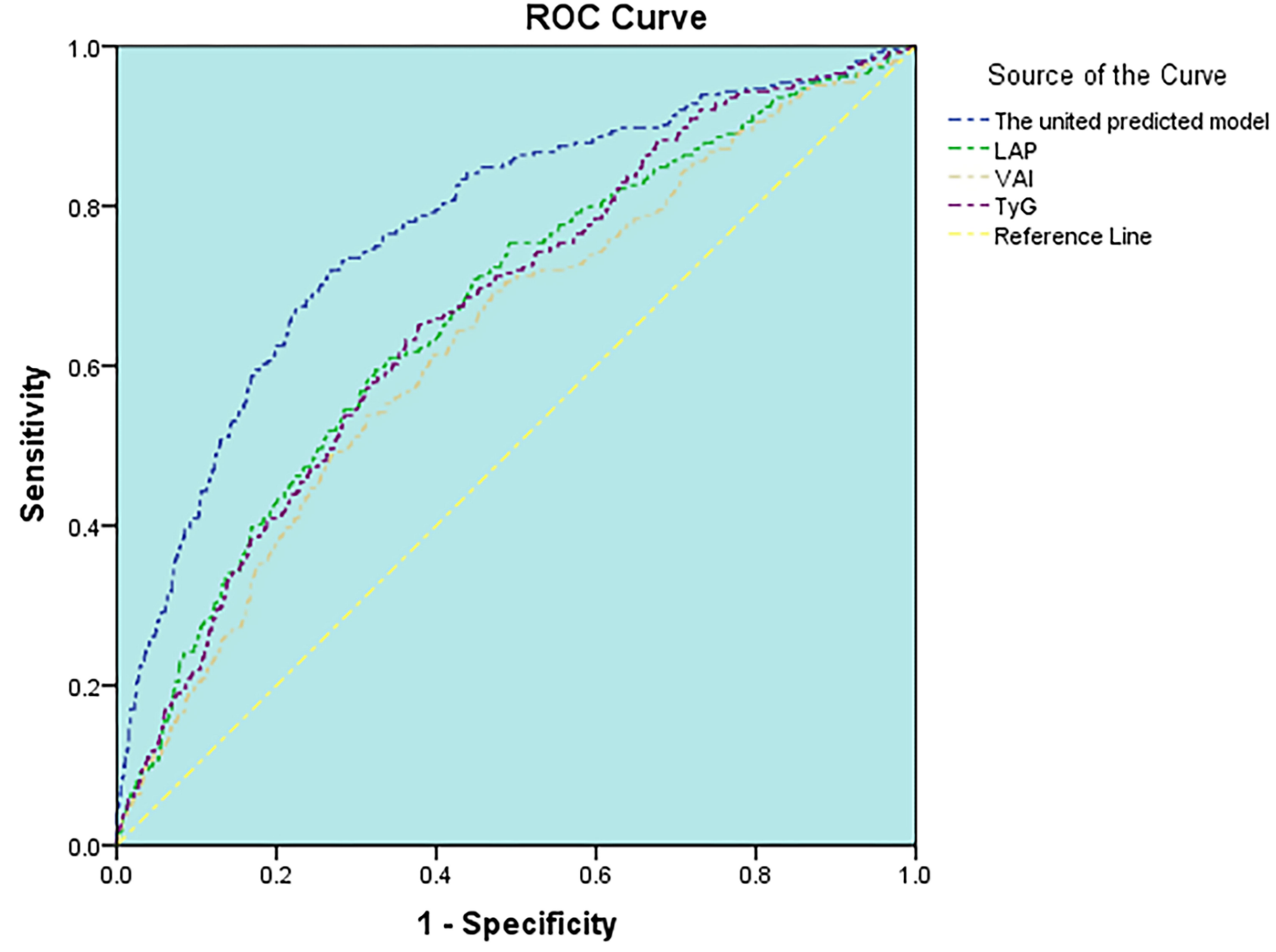

\section{Diagonal segments are produced by ties.}

Figure 2 ROC curve analysis of the value of TyG, LAPI, VAI, united predicted model for predicting CKD in females.

variables was firstly developed by Marcos amato and his team, their results showed that VAI is a valuable index of visceral adipose function. ${ }^{9}$ In a recent Korean population study, VAI was confirmed that can replace visceral CT scanning as a maker for adiposity dysfunction and lipid overaccumulation. ${ }^{41}$ LAPI includes WC and TG, similarly to VAI, and also is an effective marker for identifying excessive lipid accumulation and metabolic obesity and correlate with metabolic syndrome. ${ }^{42,43}$ Whereas TyG includes only metabolic variables (FPG and TG), and have been suggested as a biomarker of glycemic control, even better than HOMA-IR. ${ }^{44}$ Dyslipidemia and hyperglycemia are 2 basic mechanisms of insulin resistance[50]. When insulin resistance develops, the amount of fatty acids increases due to the enhancement of lipolysis and increased flux of free fatty acids into the liver cause elevated synthesis of hepatic triglyceride, forming hypertriglyceridemia. ${ }^{45,46}$ Insulin also regulates the expression and translocation of glucose transporters in different types of tissues. When insulin resistance develops, insulin insensitivity leads to the accumulation of glucose in the bloodstream, causing hyperglycemia. ${ }^{47}$ Thus, based on the above theory, TyG which estimate insulin resistance by evaluating both blood triglyceride and glucose level has potential value to predict the development of insulin resistance. ${ }^{48}$

In our study, VAI, LAPI, TyG showed a significant association with incident CKD both in males and females after adjustment for age, education, physical activity, smoking, drinking. However, when adding BMI, waist circumference, blood pressure, high-sensitivity $\mathrm{C}$ reactive protein, HDL-C, VLDL-C, LDL-C, TC to the model, there was a strong correlation between only TyG and CKD. As it is well known, waist circumference, blood pressure, 
HDL-C are parts of the diagnostic criteria of metabolic syndrome and TyG still showed strong predictive value after adjusting for these metabolic factors. According to the ROC curve, TyG showed the greater AUC in both males (AUC $=0.618 ; 95 \% \mathrm{CI}, 0.572-0.664$ ) and females (AUC $=0.670 ; 95 \%$ CI, 0.636-0.705), and LAPI also revealed greater $\mathrm{AUC}$ in females $(\mathrm{AUC}=0.670 ; 95 \% \mathrm{CI}$, 0.634-0.705). And we also found unite TyG and other traditional factors such as age, blood pressure, inflammatory factors can improve the accuracy for predicting CKD. These three novel indices seemed to be more valuable in females for predicting CKD. There are several studies found that the correlation between VAT and TG was greater in females than in males ${ }^{49}$ and the greater association between VAT and adverse metabolic outcome among females has been suggested. ${ }^{50,51}$ The mechanisms underlying gender-specific differences are not known. Different sex hormones might act on the fat distribution which subsequently affects the association between obesity and CKD. ${ }^{52}$

In conclusion, the novel combined metabolic index TyG was superior to LAPI and VAI in association with $\mathrm{CKD}$, and TyG might be a useful clinical indicator of $\mathrm{CKD}$ in the Chinese population, which is more valuable in females. Our study also proved the association between insulin resistance and renal damage and emphasized the importance of simultaneous control of blood glucose and lipids in the progression of CKD.

There are several limitations to this study. Firstly, this is a retrospective single-center cross-sectional study, the analysis of the causative effect of TyG, VAI, LAPI on CKD is limited. Secondly, another limitation of this study is the absence of other inflammation biomarkers and cytokines. Thirdly, the sample size of this study was relatively small and the sample size of males is smaller than females. Additionally, the particular TyG, VAI, LAPI cut-off points cannot use in other racial groups.

\section{Funding}

This study was supported by the "Science and Technique Program of Guangzhou" (201604020015, 2015), risk factors and prediction model of chronic kidney disease caused by metabolic syndrome: A multicentric prospective cohort study Clinical trial training project of Southern Medical University (LC2016PY047, 2016) and "The National Natural Science Foundation of China '(81873620).' South Wisdom Valley Innovative Research Team Program" (CXTD-004, 2014).

\section{Disclosure}

The authors state that there is no conflict of interest.

\section{References}

1. Matsushita K, Ballew SH, Coresh J. Cardiovascular risk prediction in people with chronic kidney disease. Curr Opin Nephrol Hypertens. 2016;25(6):518-523.

2. Webster AC, et al. Chronic kidney disease. Lancet. 2017;389 (10075):1238-1252.

3. Zhang L, Wang F, Wang L, et al. Prevalence of chronic kidney disease in China: a cross-sectional survey. Lancet. 2012;379(98 18):815-822.

4. Wang H, Zhang L, Lv J. Prevention of the progression of chronic kidney disease: practice in China. Kidney Int Suppl. 2005;94:S63-7.

5. Eknoyan G, Lameire N, Barsoum R, et al. The burden of kidney disease: improving global outcomes. Kidney Int. 2004;66(4):1310-1314.

6. Gnudi L, Coward R, Long DA. Diabetic nephropathy: perspective on novel molecular mechanisms. Trends Endocrinol Metab. 2016;27 (11):820-830.

7. DeFronzo RA, Tobin JD, Andres R. Glucose clamp technique: a method for quantifying insulin secretion and resistance. $\mathrm{Am}$ J Physiol. 1979;237(3):E214-23.

8. Muniyappa R, Lee S, Chen $\mathrm{H}$, et al. Current approaches for assessing insulin sensitivity and resistance in vivo: advantages, limitations, and appropriate usage. Am J Physiol Endocrinol Metab. 2008;294(1): E15-26.

9. Amato MC, et al. Visceral Adiposity Index: a reliable indicator of visceral fat function associated with cardiometabolic risk. Diabetes Care. 2010;33(4):920-922.

10. Wakabayashi I, Daimon T. A strong association between lipid accumulation product and diabetes mellitus in japanese women and men. $J$ Atheroscler Thromb. 2014;21(3):282-288.

11. Mazidi M, Kengne AP, Katsiki N, et al. Lipid accumulation product and triglycerides/glucose index are useful predictors of insulin resistance. J Diabetes Complications. 2018;32(3):266-270.

12. Elizalde-Barrera CI, et al. Triglycerides and waist to height ratio are more accurate than visceral adiposity and body adiposity index to predict impaired fasting glucose. Diabetes Res Clin Pract. 2019; 153:49-54.

13. Ahn N, Baumeister SE, Amann U, et al. Visceral adiposity index (VAI), lipid accumulation product (LAP), and product of triglycerides and glucose (TyG) to discriminate prediabetes and diabetes. Sci Rep. 2019;9(1):9693.

14. Brahimaj A, Rivadeneira F, Muka T, et al. Novel metabolic indices and incident type 2 diabetes among women and men: the Rotterdam Study. Diabetologia. 2019;62(9):1581-1590.

15. Nascimento-Ferreira MV, Rendo-Urteaga T, Vilanova-Campelo RC, et al. The lipid accumulation product is a powerful tool to predict metabolic syndrome in undiagnosed Brazilian adults. Clin Nutr. 2017;36(6):1693-1700.

16. Dai D, Chang Y, Chen Y, et al. Visceral adiposity index and lipid accumulation product index: two alternate body indices to identify chronic kidney disease among the rural population in Northeast China. Int J Environ Res Public Health. 2016;13:12.

17. Shi W, et al. Estimate of reduced glomerular filtration rate by triglyceride-glucose index: insights from a general Chinese population. Postgrad Med. 2019;131(4):287-294.

18. Simental-Mendia LE, Rodriguez-Moran M, Guerrero-Romero F. The product of fasting glucose and triglycerides as surrogate for identifying insulin resistance in apparently healthy subjects. Metab Syndr Relat Disord. 2008;6(4):299-304.

19. Kahn HS. The "lipid accumulation product" performs better than the body mass index for recognizing cardiovascular risk: a populationbased comparison. BMC Cardiovasc Disord. 2005;5:26. 
20. Ross R, Freeman J, Hudson R, Janssen I. Abdominal obesity, muscle composition, and insulin resistance in premenopausal women. J Clin Endocrinol Metab. 2002;87(11):5044-5051.

21. Ma YC, Zuo L, Chen JH, et al. Modified glomerular filtration rate estimating equation for Chinese patients with chronic kidney disease. J Am Soc Nephrol. 2006;17(10):2937-2944.

22. Shively CA, Register TC, Clarkson TB. Social stress, visceral obesity, and coronary artery atherosclerosis: product of a primate adaptation. Am J Primatol. 2009;71(9):742-751.

23. Yu S, Yang H, Guo X, Zheng L, Sun Y. Association between obese phenotype and mildly reduced eGFR among the General Population from Rural Northeast China. Int J Environ Res Public Health. 2016;13:6.

24. Stepien M, Stępień A, Wlazeł RN, et al. Obesity indices and adipokines in non-diabetic obese patients with early stages of chronic kidney disease. Med Sci Monit. 2013;19:1063-1072.

25. Boyko EJ, Fujimoto WY, Leonetti DL, et al. Visceral adiposity and risk of type 2 diabetes: a prospective study among Japanese Americans. Diabetes Care. 2000;23(4):465-471.

26. McLaughlin T, et al. Preferential fat deposition in subcutaneous versus visceral depots is associated with insulin sensitivity. $J$ Clin Endocrinol Metab. 2011;96(11):E1756-60.

27. Neeland IJ, Turer AT, Ayers CR, et al. Dysfunctional adiposity and the risk of prediabetes and type 2 diabetes in obese adults. JAMA 2012;308(11):1150-1159.

28. Kim SR, Yoo JH, Song HC, et al. Relationship of visceral and subcutaneous adiposity with renal function in people with type 2 diabetes mellitus. Nephrol Dial Transplant. 2011;26(11):3550-3555.

29. DeNino WF, Tchernof A, Dionne IJ, et al. Contribution of abdominal adiposity to age-related differences in insulin sensitivity and plasma lipids in healthy nonobese women. Diabetes Care. 2001;24(5):925-932.

30. Kwakernaak AJ, Zelle DM, Bakker SJ, et al. Central body fat distribution associates with unfavorable renal hemodynamics independent of body mass index. J Am Soc Nephrol. 2013;24(6):987-994

31. Roubicek T, Bartlova M, Krajickova J, et al. Increased production of proinflammatory cytokines in adipose tissue of patients with end-stage renal disease. Nutrition. 2009;25(7-8):762-768.

32. Dave N, Wu J, Thomas S. Chronic kidney disease-induced insulin resistance: current state of the field. Curr Diab Rep. 2018;18(7):44.

33. Shoelson SE, Lee J, Goldfine AB. Inflammation and insulin resistance. J Clin Invest. 2006;116(7):1793-1801.

34. Tansey JT, et al. Perilipin ablation results in a lean mouse with aberrant adipocyte lipolysis, enhanced leptin production, and resistance to diet-induced obesity. Proc Natl Acad Sci U S A. 2001;98 (11):6494-6499.

35. Becker B, Kronenberg F, Kielstein JT, et al. Renal insulin resistance syndrome, adiponectin and cardiovascular events in patients with kidney disease: the mild and moderate kidney disease study. $\mathrm{J} \mathrm{Am}$ Soc Nephrol. 2005;16(4):1091-1098.

36. Fliser D, Pacini G, Engelleiter R, et al. Insulin resistance and hyperinsulinemia are already present in patients with incipient renal disease. Kidney Int. 1998;53(5):1343-1347.
37. Kobayashi S, Maesato K, Moriya H, Ohtake T, Ikeda T. Insulin resistance in patients with chronic kidney disease. Am J Kidney Dis. 2005;45(2):275-280.

38. Fragoso A, et al. Insulin resistance as a predictor of cardiovascular morbidity and end-stage renal disease. J Diabetes Complications. 2015;29(8):1098-1104.

39. Cornier MA, Despres JP, Davis N, et al. Assessing adiposity: a scientific statement from the American Heart Association. Circulation. 2011;124(18):1996-2019.

40. Simental-Mendia LE, Rodriguez-Moran M, Guerrero-Romero F. The product of fasting glucose and triglycerides as surrogate for identifying insulin resistance in apparently healthy subjects. Metab Syndr Relat Disord. 2008;6(4):299-304.

41. Oh JY, Sung YA, Lee HJ. The visceral adiposity index as a predictor of insulin resistance in young women with polycystic ovary syndrome. Obesity (Silver Spring). 2013;21(8):1690-1694.

42. Tripathy JP, JS Thakur, G Jeet, et al. Urban rural differences in diet, physical activity and obesity in India: are we witnessing the great Indian equalisation? Results from a cross-sectional STEPS survey. BMC Public Health. 2016;16(1):816.

43. Zhang $\mathrm{XH}$, et al. Comparison of anthropometric and atherogenic indices as screening tools of metabolic syndrome in the Kazakh Adult Population in Xinjiang. Int J Environ Res Public Health. 2016;13(4):428.

44. Vasques AC, Novaes FS, de Oliveira MD, et al. TyG index performs better than HOMA in a Brazilian population: a hyperglycemic clamp validated study. Diabetes Res Clin Pract. 2011;93(3):e98-e100.

45. Mikhail N. The metabolic syndrome: insulin resistance. Curr Hypertens Rep. 2009;11(2):156-158.

46. Eckel RH, et al. The metabolic syndrome. Lancet. 2010;375 (9710):181-183.

47. Stringer DM, Zahradka P, Taylor CG. Glucose transporters: cellular links to hyperglycemia in insulin resistance and diabetes. Nutr Rev. 2015;73(3):140-154.

48. Simental-Mendia LE, Rodriguez-Moran M, Guerrero-Romero F. The product of fasting glucose and triglycerides as surrogate for identifying insulin resistance in apparently healthy subjects. Metab Syndr Relat Disord. 2008;6(4):299-304.

49. Smith SR, Lovejoy JC, Greenway F, et al. Contributions of total body fat, abdominal subcutaneous adipose tissue compartments, and visceral adipose tissue to the metabolic complications of obesity. Metabolism. 2001;50(4):425-435.

50. Kanaya AM, et al. Adipocytokines attenuate the association between visceral adiposity and diabetes in older adults. Diabetes Care. 2004;27(6):1375-1380.

51. Tanaka S, Togashi K, Rankinen T, et al. Sex differences in the relationships of abdominal fat to cardiovascular disease risk among normal-weight white subjects. Int $J$ Obes Relat Metab Disord. 2004;28(2):320-323.

52. Kahn HS, Cheng YJ. Longitudinal changes in BMI and in an index estimating excess lipids among white and black adults in the United States. Int J Obes (Lond). 2008;32(1):136-143.

Diabetes, Metabolic Syndrome and Obesity: Targets and Therapy

Dovepress

\section{Publish your work in this journal}

Diabetes, Metabolic Syndrome and Obesity: Targets and Therapy is an international, peer-reviewed open-access journal committed to the rapid publication of the latest laboratory and clinical findings in the fields of diabetes, metabolic syndrome and obesity research. Original research, review, case reports, hypothesis formation, expert opinion and commentaries are all considered for publication. The manuscript management system is completely online and includes a very quick and fair peer-review system, which is all easy to use. Visit http://www.dovepress.com/testimonials.php to read real quotes from published authors. 